\title{
THE LEONARD J. SAVAGE AWARD
}

Members of the NBER-NSF Seminar on Bayesian Inference in Econometrics and Statistics, the International Society for Bayesian Analysis, and the ASA Section on Bayesian Statistical Science are cosponsoring an annual Leonard J. Savage Award of $\$ 750$ for an outstanding doctoral dissertation in the area of Bayesian Econometrics and Statistics.

To be considered for the 1997 Savage Award, a doctoral dissertation must be submitted by the dissertation supervisor before December 31, 1997, and accompanied by a short letter from the supervisor summarizing the main results of the dissertation. Dissertations completed after January 1, 1977, are eligible to be considered for the 1997 Savage Award. An evaluation committee will be appointed by the board of the Leonard J. Savage Memorial Trust Fund (S.E. Fienberg, S. Geisser, J.B. Kadane, E.E. Leamer, J.W. Pratt, and A Zellner, Chairman) to evaluate dissertations that are submitted for the Savage award.

Dissertations and supporting letters should be sent to Professor Arnold Zellner, Graduate School of Business, University of Chicago, 1101 East 58th Street, Chicago, IL 60637, USA.

The 1996 winners will be announced in the near future. The co-winners of the 1995 Savage Award are Alyson Wilson for her thesis, "Statistical Models for Shapes and Deformations," completed at Duke University under the direction of Valen Johnson, and Christopher Carter for his thesis, "Markov Chain Monte Carlo Methods for State Space Models," completed at the University of New South Wales, Australia, under the direction of Robert Kohn.

The following candidates were accorded Honorable Mention: Ming-Hui Chen, "Monte Carlo Markov Chain Sampling for Evaluating Multivariate Integrals with Applications to Bayesian Computation," completed at Purdue University under the direction of James Berger, and Simon J. Godsill, "Bayesian Enhancement of Speech and Audio Signals in the Presence of Both Impulsive and Background Noise," completed at Cambridge University under the direction of P.J.W. Rayner. 